Provided for non-commercial research and education use. Not for reproduction, distribution or commercial use.

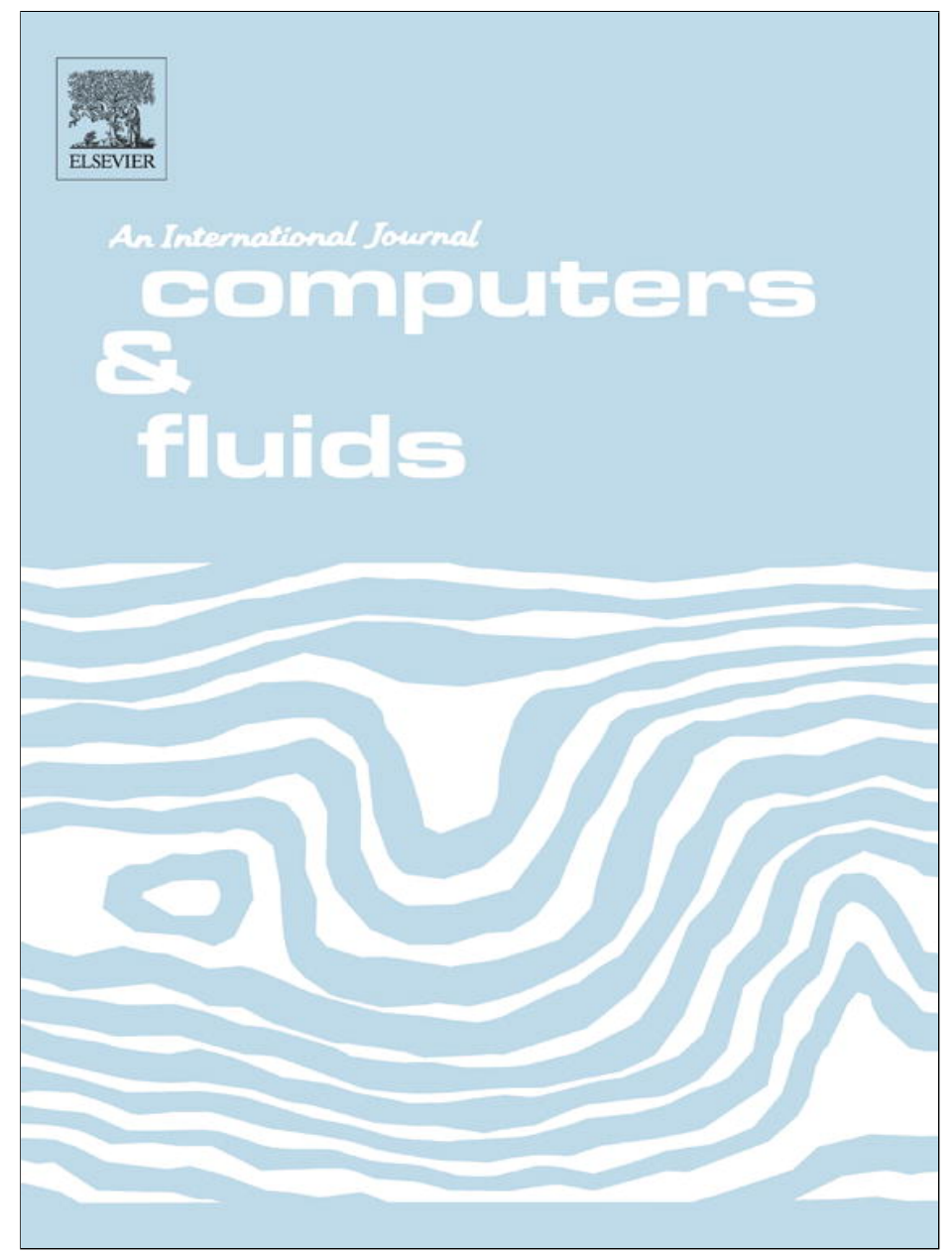

This article appeared in a journal published by Elsevier. The attached copy is furnished to the author for internal non-commercial research and education use, including for instruction at the authors institution and sharing with colleagues.

Other uses, including reproduction and distribution, or selling or licensing copies, or posting to personal, institutional or third party websites are prohibited.

In most cases authors are permitted to post their version of the article (e.g. in Word or Tex form) to their personal website or institutional repository. Authors requiring further information regarding Elsevier's archiving and manuscript policies are encouraged to visit:

http://www.elsevier.com/authorsrights 


\title{
Comparative assessment of Volume-of-Fluid and Level-Set methods by relevance to dendritic ice growth in supercooled water
}

\author{
P. Rauschenberger ${ }^{\mathrm{a}, *}$, A. Criscione ${ }^{\mathrm{b}}$, K. Eisenschmidt $^{\mathrm{a}}$, D. Kintea ${ }^{\mathrm{b}}, \mathrm{S}$. Jakirlić ${ }^{\mathrm{b}, \mathrm{d}, *}, \check{Z}$. Tuković $^{\mathrm{c}}$ \\ I.V. Roisman ${ }^{\text {b,d }}$, B. Weigand ${ }^{\mathrm{a}}$, C. Tropea ${ }^{\mathrm{b}, \mathrm{d}}$
}

${ }^{a}$ Institute of Aerospace Thermodynamics, University of Stuttgart, Germany

${ }^{\mathrm{b}}$ Institute for Fluid Mechanics and Aerodynamics, Technische Universität Darmstadt, Germany

${ }^{\mathrm{c}}$ Department of Mechanical Engineering and Naval Architecture, University of Zagreb, Croatia

${ }^{\mathrm{d}}$ Center of Smart Interfaces, Technische Universität Darmstadt, Germany

\section{A R T I C L E I N F O}

\section{Article history:}

Received 25 December 2012

Received in revised form 13 March 2013

Accepted 15 March 2013

Available online 27 March 2013

\section{Keywords:}

Supercooled water

Solidification

Direct numerical simulation

Level-Set

Volume-of-Fluid

\begin{abstract}
A B S T R A C T
Two relevant computational models, one relying on a Level-Set approach, the other one on a Volume-ofFluid tracking procedure with piecewise linear interface reconstruction, are comparatively assessed in terms of their capability to simulate crystallization of supercooled water. The models are preliminary validated by computing a one-dimensional freezing front propagation for which an analytic solution exists. Afterwards, the tip velocity of two-dimensional dendrites growing in supercooled water is determined and compared with corresponding experimental results and theoretical predictions in the range of supercooling between $1 \mathrm{~K}$ and $30 \mathrm{~K}$. Present modeling results following closely both the underlying theory and experimental findings show very good mutual agreement.
\end{abstract}

(c) 2013 Elsevier Ltd. All rights reserved.

\section{Introduction}

Droplet dynamics problems under extreme boundary conditions play an important role in a number of technical and natural processes. In the framework of the collaborative research center SFB-TRR 75 (Droplet Dynamics under Extreme Ambient Conditions) relevant problems are studied in detail (see e.g. a project overview of Weigand and Tropea [1]). One of the most frequently encountered freezing processes involving droplets is certainly the formation of an ice-layer on an aircraft wing during its collision with supercooled droplets. This and the process of the ice-crystal growth in clouds are studied in different sub-projects of the SFBTRR 75. For these two fundamentally different applications two different numerical codes have been used, both based on the finite volume method. For the computational study of crystal growth processes in clouds, the in-house code FS3D (Free Surface 3D) was chosen, utilizing advantage of an orthogonal Cartesian mesh. The formation of an ice-layer by drop impact onto an aircraft wing occurs in a more complex geometrical environment. For such an

\footnotetext{
* Corresponding authors. Address: Institute for Fluid Mechanics and Aerodynamics, Technische Universität Darmstadt, Germany. Tel.: +49 6151163554; fax: +496151164754 (S. Jakirlić).

E-mail addresses: philipp.rauschenberger@itlr.uni-stuttgart.de (P. Rauschenberger),s.jakirlic@sla.tu-darmstadt.de (S. Jakirlić).
}

application the open source software OpenFOAM ${ }^{\circledR}$ was selected. The two codes use different front tracking methods. In conjunction with the OpenFOAM ${ }^{\circledR}$ code an appropriate Level-Set approach has been proposed and implemented, whereas the FS3D code benefits from a Volume-of-Fluid (VOF) method with Piecewise Linear Interface Calculation (PLIC).

Accordingly, the objective of the present work is a comparative validation of both tools on the basis of some generic configurations. Two cases of particular interest are the 1-D ice-layer growth for which an analytic solution was derived [2] and a 2-D case, where the tip velocity of parabolic-shaped dendrites is validated along with the experimental data of [3-11]. Furthermore, the predictive performances of the two front capturing methods are mutually compared. The numerical simulations have been performed under conditions where pure water may retain its liquid state at temperatures as low as approximately $-35{ }^{\circ} \mathrm{C}$ before homogeneous nucleation starts. Correspondingly the water is in a metastable state. In the case of heterogeneous nucleation, the critical temperature is respectively higher. With the FS3D code the intention is to perform direct numerical simulations of the entire relevant scale span characterizing the droplet freezing process. At the microscale, a nucleation model initiates the ice seed in a potentially deformed liquid droplet; afterwards the phase growth starts within the droplet on a mesoscale. On the macroscale, the config- 
urations including droplet-droplet and droplet-ice interactions will be studied. The droplet-wall interaction, ice-wall interaction and ice growth at walls as encountered during wing icing will be studied using the OpenFOAM ${ }^{\circledR}$ software. Here the correct capturing of the hydrodynamics of the supercooled liquid particle approaching the wall and its spreading on the wall is of decisive importance. The OpenFOAM ${ }^{\circledR}$ code enables handling of arbitrarily complex wall geometries.

A complexity arising from the process of ice seed growing in liquid water that is cooled down to temperatures below its equilibrium solidification temperature is closely connected to an unstable freezing surface. In this process supercooling acts as the driving force towards the phase growth; the interface is instable [12-14] and small perturbations at the ice surface lead to a bump-like expansion of ice propagating into the liquid which experiences a steeper temperature gradient. This enhances locally the ice growth and the formation of dendrites. As described in detail in Section 2, this effect is attenuated by the product of surface tension and the local curvature. For that, an exact representation of the interface morphology is indispensable. Accordingly, the direct numerical solution of such a process corresponding to the time-dependent Stefan problem represents a great challenge. An appropriate front tracking method accounting for the moving solid-liquid interface is required. Over the last decades various computational models have been applied for the simulation of dendritic growth.

Juric and Tryggvason [15] have presented a front-tracking method on a fixed Cartesian grid for the simulation of dendritic solidification in two dimensions. The information exchange between the Eulerian and Lagrangian interface points was accomplished by applying the Immersed Boundary Method (IBM) [16]. The heat conduction equation is discretized using finite differences. An iterative scheme for the interface velocity determination serving for the temperature distribution satisfies the Dirichlet boundary condition at the interface. The method was extended to include advection [17] and three-dimensionality [18]. Udaykumar et al. [19] presented an approach similar to that of Juric and Tryggvason [15], however, the interface velocity was determined directly from the Stefan condition imposing temperature at the sharp interface. Beckermann et al. [20] introduced a diffuse interface model accounting for advection within the liquid phase. They conducted 2-D simulations of dendritic growth of an initially round particle with and without melt advection; the study analyzed the tip velocities and radii upstream, downstream and normal to the flow direction. Karagadde et al. [21] presented recently a VOF-IBM-enthalpy approach in conjunction with a 2D simulation of dendritic growth including advection. They applied the enthalpy method utilizing an iterative scheme to update the liquid fraction in a cell due to the enthalpy change. Liquid cells next to the solid ones are seeded with small amounts of solid to initiate growth. They adopted the IBM method to solve the liquid flow including a solid body. The solid phase is advected using the VOF-technique with interface reconstruction. Level-Set methods have been increasingly applied to several problems involving moving boundaries [22,23] and crystallization [24,25] exhibiting good predictive performance in returning qualitative features of the dendrites.

The paper starts with an outline of the underlying physics and the mathematical description of the problem considered. In Section 3, the numerical frameworks pertinent to both codes FS3D and OpenFOAM ${ }^{\circledR}$ is explained. The computational models' validation by means of an analytic solution of a one-dimensional solidification problem as well as the results comparison with respect to the dendrite tip velocities as predicted by the universal law of dendritic growth of Langer and Müller-Krumbhaar [26] are illustrated in Section 4.

\section{Physical formulation}

For incompressible flow and constant fluid properties, the momentum equations are decoupled from the energy equation. The problem considered is characterized by small Eckert numbers $E c=\mathbf{u}^{2} /\left(c_{p} \Delta T\right) \ll 1$ due to low velocity conditions. Here $\mathbf{u}$ denotes the velocity vector, $c_{p}$ the specific heat capacity and $T$ the temperature. This allows the viscous heating to be neglected with the thermal energy equation governing the temperature field. Since the densities of water $\rho_{l}$ and ice $\rho_{s}$ differ only slightly, equal density $\rho$ is used for both phases.

We use the index $l$ for liquid and the index $s$ for solid; $i=(s, l)$ if solid or liquid appear exclusively in an equation valid for both phases. The thermal energy equation governing the dynamics of the temperature field reads [27]

$\frac{\partial\left(\rho c_{p, i} T_{i}\right)}{\partial t}+\nabla \cdot\left(\rho c_{p, i} T_{i} \mathbf{u}\right)=\nabla \cdot\left(k_{i} \nabla T_{i}\right)+\dot{q}_{i}^{\prime \prime \prime}$,

where $k$ denotes the heat conductivity and $\dot{q}^{\prime \prime \prime}$ is a volumetric heat source. Eq. (1) is solved in both phases separately. The temperature fields of solid and liquid are connected by the Stefan condition in all interface cells. Assuming local equilibrium, it states that the latent heat released during phase change must be conducted away from the interface in both phases; the corresponding mathematical formulation reads

$\dot{m}^{\prime \prime}\left[L-\left(c_{p, l}-c_{p, s}\right)\left(T_{m}-T_{\Gamma}\right)\right]=-\lambda_{l} \nabla T_{l} \cdot \mathbf{n}_{\Gamma}+\lambda_{s} \nabla T_{s} \cdot \mathbf{n}_{\Gamma}$

Here $\mathbf{n}_{\Gamma}$ is the interface normal vector, $T_{\Gamma}$ the interface temperature and $T_{m}$ the melting temperature of water. The second term in square brackets accounts for the difference in the heat capacities of water and ice [28]. The mass flux per unit area can also be expressed in terms of the interface velocity: $\dot{m}^{\prime \prime}=\rho\left|\mathbf{V}_{\Gamma}\right| . T_{\Gamma}$ is the fusion temperature of water on curved interfaces. For normal pressure and flat interfaces $T_{\Gamma}$ is equal to $T_{m}=273.15 \mathrm{~K}$. In contrast to supercooling, which is the driving force for dendritic growth, the surface tension acts as a counterforce, because it reduces the local supercooling on curved interfaces. These two opposed mechanisms drive other effects like tip splitting and sidebranching and determine consequently the crystal morphology. The relation between surface tension and melting temperature depression can be described by the Gibbs-Thomson relation [28]

$T_{\Gamma}=T_{m}\left(1-\frac{\gamma \kappa}{\rho L}\right)+\frac{\left(c_{p, l}-c_{p, s}\right) T_{m}}{L}\left[T_{\Gamma} \ln \left(\frac{T_{\Gamma}}{T_{m}}\right)+\left(T_{m}-T_{\Gamma}\right)\right]$,

where the curvature $\kappa$ of the interface is defined as the sum of the reciprocals of the two main radii of curvature; $\gamma$ denotes the surface tension. Hence, there are two boundary conditions at the solid-liquid interface. The first one is a Dirichlet boundary condition and determines the interface temperature according to the local curvature. The second one is the Stefan condition, implying the latent heat release being transferred by conduction during phase change into both the solid phase and the liquid phase.

\section{Numerical schemes}

For phase change problems, in particular water-ice systems, it is of great importance to reproduce the morphology and especially the curvature of the ice particle. This means that the interface between solid and liquid must be well resolved and sharp. Existing methods for the simulation of free surfaces can be classified into two groups [29], namely:

- Interface-tracking methods,

- Interface-capturing methods. 
Interface-tracking methods apply boundary-fitted grids, while interface-capturing methods work on a fixed grid. The VOF method [30] and the Level-Set method [31,32] are an example of the latter. In this section, the two approaches, one using VOF, the other one Level-Set, are presented in the context of solidification problems.

\subsection{Volume-of-Fluid (VOF) method within the FS3D code}

In the FS3D code, the VOF method [30] is used to capture the interface between different phases. Presently the scalar field variable $f$ identifies the volume fraction of the solid, i.e. the ice phase within a cell. Here, it is defined as

$f(\mathbf{x}, t)= \begin{cases}0 & \text { in the fluid, } \\ {[0 ; 1]} & \text { at theinterface, } \\ 1 & \text { in the solid. }\end{cases}$

The Piecewise Linear Interface Calculation (PLIC) algorithm [33] is applied to reconstruct a plane separating the two phases in the cells comprising the interface; the plane is perpendicular to the local interface normal vector $\mathbf{n}_{\Gamma}$ representing actually the negative gradient of the volume fraction $f$. It is computed in a three-dimensional case from a 27-point stencil taking into account the VOF distribution in neighboring cells pointing into the direction from the solid to the liquid phase. With knowledge of the interface position, the volume fluxes needed for advection and for phase transition can be calculated. Advantages of the VOF/PLIC approach are volume conservation and the capability to track convoluted surfaces. A drawback is that for large curvatures structures smaller than the grid size may not be resolved [34]. The motion of the ice particle resembles a rigid body motion as described in Rauschenberger et al. [35]. However, the configurations considered in the present work do not require the fluid advection or motion of the solid ice phase to be accounted for.

\subsubsection{Thermal energy equation}

As previously discussed advection phenomena are not represented in the present results and therefore are not considered in the equation for thermal energy. According to the two-scalar approach the thermal energy equation is solved independently in both phases [36] and thus, averaged fluid properties are not needed. The Stefan condition connects both temperature fields.

The thermal energy source is computed from Eq. (2) as the sum of the heat fluxes towards either side of the interface. Knowing it, the mass source is extracted by division with the term in square brackets. This means, the temperature gradients in direction of the interface normal are needed. Hence, the Stefan condition can be reduced to a local $1 \mathrm{D}$ problem:

$\dot{q}_{s}^{\prime \prime}=\left.\lambda_{s} \nabla T\right|_{s} \cdot \mathbf{n}_{\Gamma}=\lambda_{s} \frac{T_{\Gamma}-T_{v, s}}{d}$,

$\dot{q}_{l}^{\prime \prime}=\left.\lambda_{l} \nabla T\right|_{l} \cdot \mathbf{n}_{\Gamma}=\lambda_{l} \frac{T_{v, l}-T_{\Gamma}}{d}$.

The interface temperature $T_{\Gamma}$ is given implicitly in Eq. (3). The temperatures $T_{v, s}$ and $T_{v, l}$ are determined at the fixed distance $d$ from the center of the PLIC-reconstructed interface area by a trilinear interpolation, where $d$ is 1.2 times the root mean square of the cell-face lengths of the local grid cell, cp. [17,18]. A gradient of second-order accuracy is realized with the help of a second temperature at the distance $2 d$. The volumetric heat fluxes $\dot{q}_{i}^{\prime \prime \prime}$ are obtained by multiplying the individual fluxes per surface area $\dot{q}_{i}^{\prime \prime}$ with the surface density $a_{\Gamma}$, representing the ratio of surface area to cell volume in an interface cell. The surface area is the exact value originating from the PLIC reconstruction. The heat conduction equation is solved implicitly; the derivatives are approximated by sec- ond-order accurate central differencing schemes. Furthermore, in order to obtain a stable solution when phase change is involved, we limited the time step to

$\Delta t \leqslant \frac{h_{\min }^{2}}{2 \alpha_{\max }}$,

where $\alpha_{\max }$ is the maximum thermal diffusivity (either of the fluid or of the solid) and $h_{\min }$ the representative minimum grid spacing. The heat sources $\dot{q}_{l}^{\prime \prime \prime}$ and $\dot{q}_{s}^{\prime \prime \prime}$ are added in the liquid phase and the solid phase respectively; accordingly, the temperature at the time step $(n+1)$ corresponding to phase $i$ reads

$T_{i}^{n+1}=T_{i}^{n}+\frac{\Delta t}{\left(\rho\left(f^{n}\right) c_{p, i}\left(f^{n}\right)\right)^{n+1}}\left[\nabla \cdot\left[\lambda_{i}\left(f^{n}\right) \nabla T_{i}^{n}\right]+\dot{q}_{i}^{\prime \prime \prime}\right]$.

The volumetric mass source obtained from the sum of the two heat fluxes

$\dot{m}^{\prime \prime \prime}=\frac{\dot{q}_{s}^{\prime \prime \prime}-\dot{q}_{l}^{\prime \prime \prime}}{L-\left(c_{p, l}-c_{p, s}\right)\left(T_{m}-T_{\Gamma}\right)}$

is superimposed to the VOF fraction field $f^{n}$ :

$f^{n+1}=f^{n}+\Delta t \frac{\dot{m}^{\prime \prime \prime}}{\rho}$

The internal energy of the mass that solidifies must be added to the thermal energy of the ice phase in all interface cells. The phase change takes place at temperature $T_{\Gamma}$. Thus, in the case of freezing, the solid temperature must be updated according to

$T_{s}^{n+1}:=\frac{1}{f^{n+1}}\left[\left(f^{n+1}-\Delta t \frac{\dot{m}^{\prime \prime \prime}}{\rho}\right) T_{s}^{n+1}+\Delta t \frac{\dot{m}^{\prime \prime \prime}}{\rho} T_{\Gamma}\right]$,

and in the case of melting, the liquid temperature is updated appropriately

$T_{l}^{n+1}:=\frac{1}{1-f^{n+1}}\left[\left(1-\left(f^{n+1}-\Delta t \frac{\dot{m}^{\prime \prime \prime}}{\rho}\right)\right) T_{l}^{n+1}-\Delta t \frac{\dot{m}^{\prime \prime \prime}}{\rho} T_{\Gamma}\right]$.

\subsection{Level-Set method within the OpenFOAM ${ }^{\circledR}$ code}

The Level-Set method has been formulated and implemented into the open source software OpenFOAM ${ }^{\circledR}$ in order to track the interface between the solid and the liquid region. In the following, an overview of the new Level-Set implementation is given. For a detailed description we refer to Criscione et al. [37].

The basic idea behind the Level-Set method is to track implicitly the moving boundary. Therefore, a Level-Set field $\Phi$ is defined in the entire domain. The liquid and solid phases are denoted by the points fulfilling the criteria $\Phi>0$ and $\Phi<0$, respectively. The interface coincides with the iso-contour of the zero Level-Set function $\Phi=0$. Presently, a signed distance function is used for the outer Level-Set field [38]. A signed distance function is associated with that value of the outer Level-Set field representing the shortest distance to the interface. The sign denotes on which side of the interface the certain point is located. The Level-Set equation

$\frac{\partial \Phi}{\partial t}+\mathbf{V}_{\Gamma} \cdot \nabla \Phi=0$

is used to move the interface according to the interfacial velocity $\mathbf{V}_{\Gamma}$, which depends upon the heat removal from the interface. This velocity can be obtained from the heat balance around the interface, Eq. (2). In order to compute $\mathbf{V}_{\Gamma}$ from this equation, the temperature at the interface $T_{\Gamma}$ defined by Eq. (3) is calculated. Using $T_{\Gamma}$, the temperature gradient into the solid and the liquid region is determined yielding finally $\mathbf{V}_{\Gamma}$. In order to obtain the new position of the interface for the next time step, the interfacial velocity is extended in the normal-to-interface direction within a narrow band 
around the interface. The width of the narrow-band $w_{n b}$ is calculated at each time step as

$w_{n b}=\frac{1}{\kappa_{\max }}$,

where $\kappa_{\max }$ is the maximum curvature of the interface. This criterion is introduced to avoid overlapping of the normal interface velocity extension. The extended velocity field, $\mathbf{v}_{e}$, is employed in a continuing step to calculate the new interface position by solving

$\Phi^{n+1}=\Phi^{n}-\mathbf{V}_{e} \cdot \nabla \Phi^{n} \Delta t$

where the index $n$ represents the current time step. The time step $\Delta t$ is adaptively corrected using the Courant-Friedrichs-Lewy (CFL) condition with $C o \leqslant 0.5$. Fig. 1 shows the extended velocity within the narrow band. After the update of the interface position, the Level-Set function is re-initialized by solving the equation

$\frac{\partial \Phi}{\partial \tau}-|\nabla \Phi|=-1$,

with the fictitious time $\tau$. The latter equation is to be solved until a steady-state solution is achieved. The fictitious time step is defined as follows:

$\Delta \tau \leqslant \frac{\operatorname{Co\Delta x}}{\max \left(\left|\mathbf{V}_{e}\right|\right)}$,

where the Courant number is set to $C o \leqslant 0.5$.

\subsubsection{Thermal energy equation}

The heat transfer equation (cf. Eq. (1)) neglecting advection and the volumetric heat source is solved in the liquid region

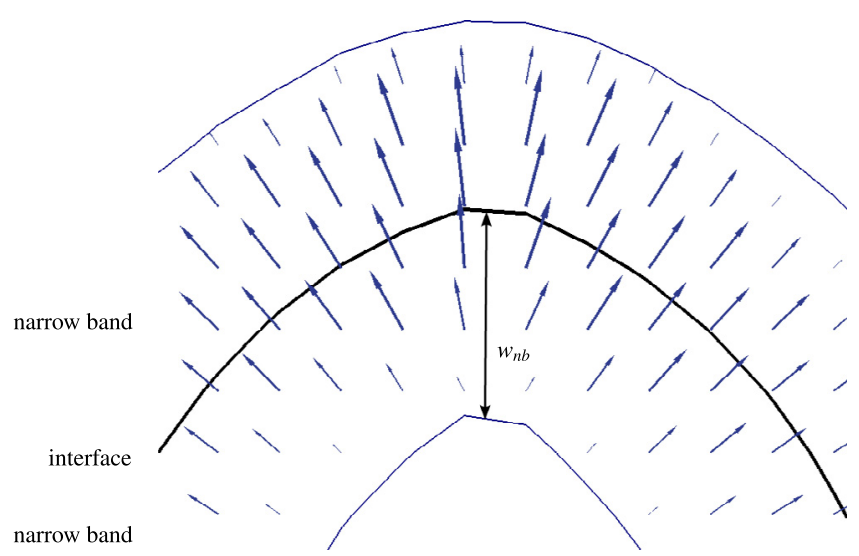

Fig. 1. Extended velocity $\mathbf{V}_{e}$ within the narrow band. independently of the solution procedure for the solid region. The computational domain is decomposed into two different sub-domains which are separated by a set of faces (ghost faces), Fig. 2. A fictitious temperature is imposed onto the ghost faces as Dirichlet boundary condition for both temperature fields, in the liquid and in the solid phase. This fictitious temperature is obtained by a linear interpolation/extrapolation using two following interpolation points: the temperature $T_{\Gamma}$ at the interface corresponding to the zero Level-Set function and the temperature value at the adjacent cell center. Imposing a fictitious temperature on the ghostfaces, a kind of immersed boundary condition is imposed which represents the influence of the moving interface on the temperature field. This is done for both regions. The equation of thermal energy is solved subsequently. In the last step, the two temperature fields are reassembled to obtain the solution within the whole domain.

\subsection{Main differences between Volume-of-Fluid and Level-Set}

The VOF method introduces an additional variable that indicates the fraction of one of the two phases within a grid cell, whereas the Level-Set is a signed distance function taking zero value at the location of the interface, with the sign of the Level-Set variable denoting the appropriate phase. The Level-Set variable varies smoothly across the interface, while VOF is discontinuous. Both methods are capable of reproducing the interface sharply. However, in the case of the VOF method, this is only true, if the interface is reconstructed in a certain manner (for instance using PLIC in the present approach). The VOF method is inherently mass-conservative, whereas the Level-Set function must be re-initialized; the latter is presently done after every time step. Furthermore, the mass conservation issue associated with the Level-Set method could be a problem if densities of phases were different to a larger extent. This is, however, not the case in the present work. Accordingly, the mass conservation issue does not impact the Level-Set implementation. With respect to the phase change, the main difference between the two presently employed schemes is the advancement of the interface. The VOF variable is updated with the mass source computed from the Stefan condition at the interface. In contrast, the Level-Set variable is moved according to the velocity corresponding to the ratio of the mass source to the ice density.

For simpler geometries meshed by an orthogonal Cartesian grid the VOF implementation is a suitable and fast tool and can be successfully used for the fundamental research involving freezing process in single droplets and interactions between a few particles. Since drops are surrounded by air, large density gradients of the order of 1000 are encountered. For problems involving complex

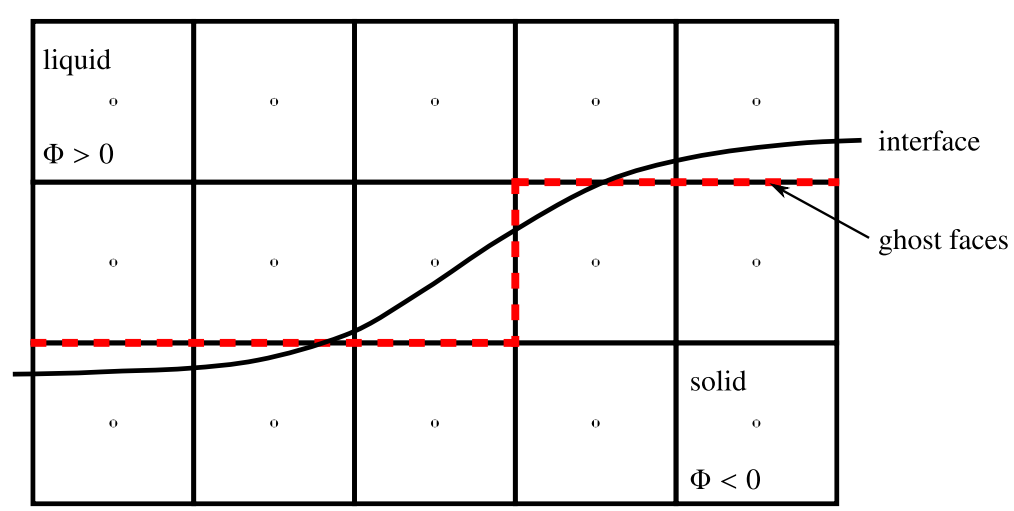

Fig. 2. Illustration of ghost faces. 
geometries with curved walls, as e.g. encountered in the case of the freezing process at an aircraft wing, the Level-Set implementation can be appropriately applied.

\section{VOF and Level-Set models validation}

A comparative assessment of the predictive capabilities of both models with respect to the results quality is performed by computing two generic configurations involving water freezing processes characterized by increasing complexity of the interface shape separating two phases.

\subsection{One-dimensional ice layer growth}

The models validation is performed preliminary by considering the phase change occurring in a one-dimensional problem of pure heat conduction for which an analytic solution is known in literature (see Carslaw and Jaeger [2]). The computational domain accommodates a flat ice front advancing in the positive $x$-direction (see Fig. 3); it is bounded by a flat solid wall on the left hand side and extends to infinity on the right hand side.

The problem considered is described by the heat conduction equation reduced to the form corresponding to a one-dimensional problem:

$0<x<X(t), \quad t>0: \quad \frac{\partial T}{\partial t}=\alpha_{s} \frac{\partial^{2} T}{\partial x^{2}}$,

$x>X(t), \quad t>0: \quad \frac{\partial T}{\partial t}=\alpha_{l} \frac{\partial^{2} T}{\partial x^{2}}$,

where $X(t)$ denotes the interface position and $\alpha_{i}=k_{i} /\left(\rho_{i} c_{p, i}\right)$ is the thermal diffusivity of the respective phase. At the interface, the Stefan condition holds, see Eq. (2). The constant temperature $T_{\Gamma}=T_{m}=273.15 \mathrm{~K}$ was set at the interface in its entirety (complying with a flat interface), hence the terms accounting for the difference in heat capacities disappear. The density of water and ice is assumed to be equal. Initial and boundary conditions are:

$t=0: \quad T(x, 0)=T_{\infty}<T_{m}, \quad X(0)=0$,

$x=0: \quad T(0, t)=T_{w}<T_{m}$,

$x \rightarrow \infty: \quad \lim _{x \rightarrow \infty} T(x, t)=T_{\infty}$,

where $T_{w}=263.15 \mathrm{~K}$ and $T_{\infty}=243.15 \mathrm{~K}$. The analytic solution reported in Carslaw and Jaeger [2] reads:

$X(t)=2 \beta \sqrt{\alpha_{s} t}$

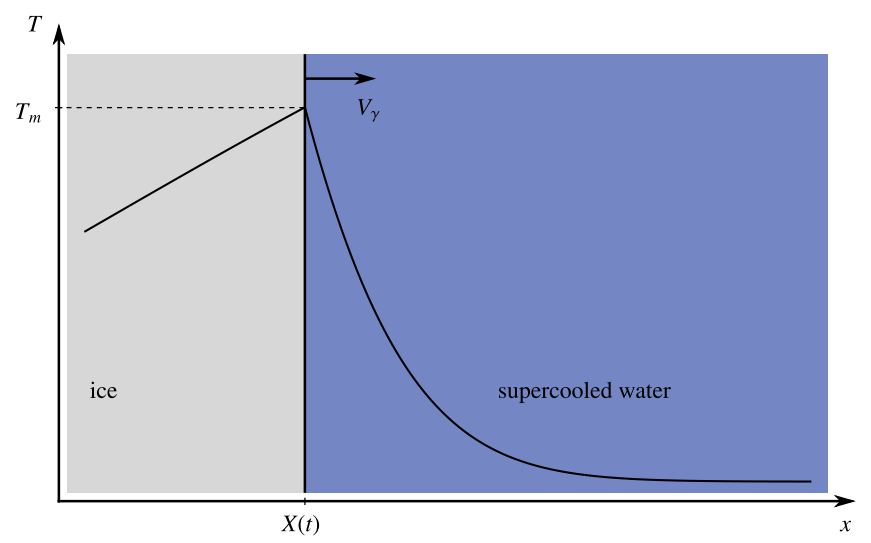

Fig. 3. Computational setup of the one-dimensional freezing process governed by heat conduction in solid and liquid phase.
Table 1

Physical properties of water at $243.15 \mathrm{~K}$ and ice at $263.15 \mathrm{~K}$.

\begin{tabular}{llllll}
\hline & $T(\mathrm{~K})$ & $\rho\left(\mathrm{kg} / \mathrm{m}^{3}\right)$ & $L(\mathrm{~kJ} / \mathrm{kg})$ & $\lambda(\mathrm{W} /(\mathrm{m} \mathrm{K}))$ & $c_{p}(\mathrm{~kJ} / \mathrm{kg} \mathrm{K})$ \\
\hline Water & 243.15 & 1000.0 & 333.6 & 0.4829 & 4.864 \\
Ice & 263.15 & 1000.0 & - & 2.319 & 2.030 \\
\hline
\end{tabular}

$0<x<X(t), \quad t>0: \quad T(x, t)=T_{w}+\left(T_{m}-T_{w}\right) \frac{\operatorname{erf}\left(\frac{x}{2 \sqrt{\alpha_{s}} t}\right)}{\operatorname{erf}(\beta)}$

$x>X(t), \quad t>0: \quad T(x, t)=T_{\infty}+\left(T_{m}-T_{\infty}\right) \frac{\operatorname{erfc}\left(\frac{x}{2 \sqrt{\alpha_{l} t}}\right)}{\operatorname{erfc}(v \beta)}$

The transcendental equation

$\beta \sqrt{\pi}=\frac{S t_{s}}{\exp \left(\beta^{2}\right) \operatorname{erf}(\beta)}+\frac{S t_{l}}{v \exp \left(v^{2} \beta^{2}\right) \operatorname{erfc}(v \beta)}$

must be solved for $\beta$. St $t_{i}$ represents the Stefan number and $v$ the ratio of thermal diffusivities:

$S t_{l}=\frac{c_{p, l}\left(T_{m}-T_{\infty}\right)}{L}, \quad S t_{s}=\frac{c_{p, s}\left(T_{m}-T_{w}\right)}{L}, \quad v=\sqrt{\frac{\alpha_{s}}{\alpha_{l}}}$.

The physical properties used in both the analytical and the numerical solution are summarized in Table 1. Evaluation of Eq. (23) yields $\beta \approx 0.2685$

The numerical grid utilized in the computations performed by both codes FS3D and OpenFOAM ${ }^{\circledR}$ is arranged as follows: the half region on the left-hand side, where the temperature field exhibits significant changes, is meshed by an equidistant grid with the respective grid spacing $h$; the value of the latter can be extracted from Figs. 4-6. On the right-hand side the grid resolution is appropriately coarsened in a non-uniform manner implying a grid spacing increase by a constant value between two consecutive cells. In Fig. 4, the temperature distribution for solid and liquid obtained by using the VOF method in conjunction with the finest numerical grid is plotted as a function of time and space. The computationally obtained dependencies are almost coincident with the analytical solution. The results displayed in Fig. 4 confirm that the computational domain is large enough (within the entire time interval considered) to assure that the heat conducted away from the interface in the liquid does not influence the boundary.

According to the analytical solution (Eq. (20)), the position of the interface exhibits a square-root dependency on time. The high growth velocity (corresponding to a steep gradient of the ice front position) is caused by a steep temperature gradient at the initial time instant. These gradients gradually weaken, in accordance with a flattened velocity and temperature evolution, as latent heat was conducted into the liquid and solid phase. The solid-liquid interface position evaluated computationally by both VOF and LevelSet methods is compared with the analytical solution in Fig. 5a and $b$ in terms of different grid resolutions. The results obtained clearly converge towards the analytical solution. The deviation of the phase front position obtained by using FS3D at $t=0.5 \mathrm{~s}$ is $3.6 \%$ on the coarsest grid and $0.3 \%$ on the finest one. Corresponding deviations obtained by using the OpenFOAM ${ }^{\circledR}$ code are $5.7 \%$ and $1.4 \%$ respectively.

Fig. 6 displays the comparison of the temperature development as functions of the $x$-coordinate at three selected time instants ( $t=0.1 \mathrm{~s}, 0.3 \mathrm{~s}$ and $0.5 \mathrm{~s}$ ) obtained by the VOF (FS3D code) method using the finest grid resolution. The results obtained by using the Level-Set (OpenFOAM ${ }^{\circledR}$ code) method (not shown here) follow closely the VOF ones. One can conclude that both methods are capable to perfectly reproduce, temporally and spatially, the analytically determined temperature evolution. 


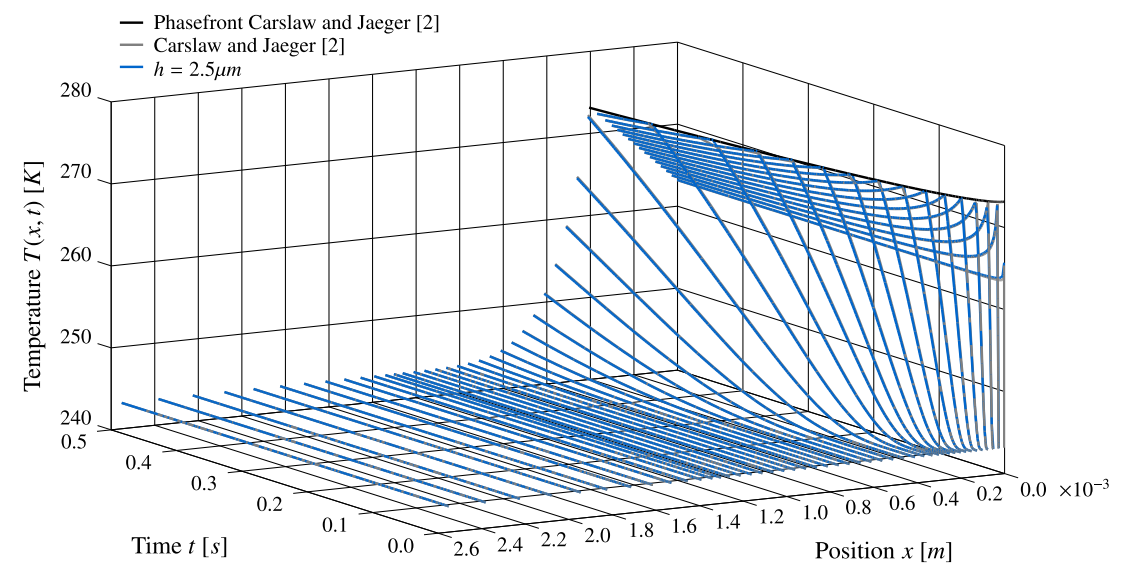

Fig. 4. Temperature distribution as function of time and $x$-coordinate obtained by the present VOF method.

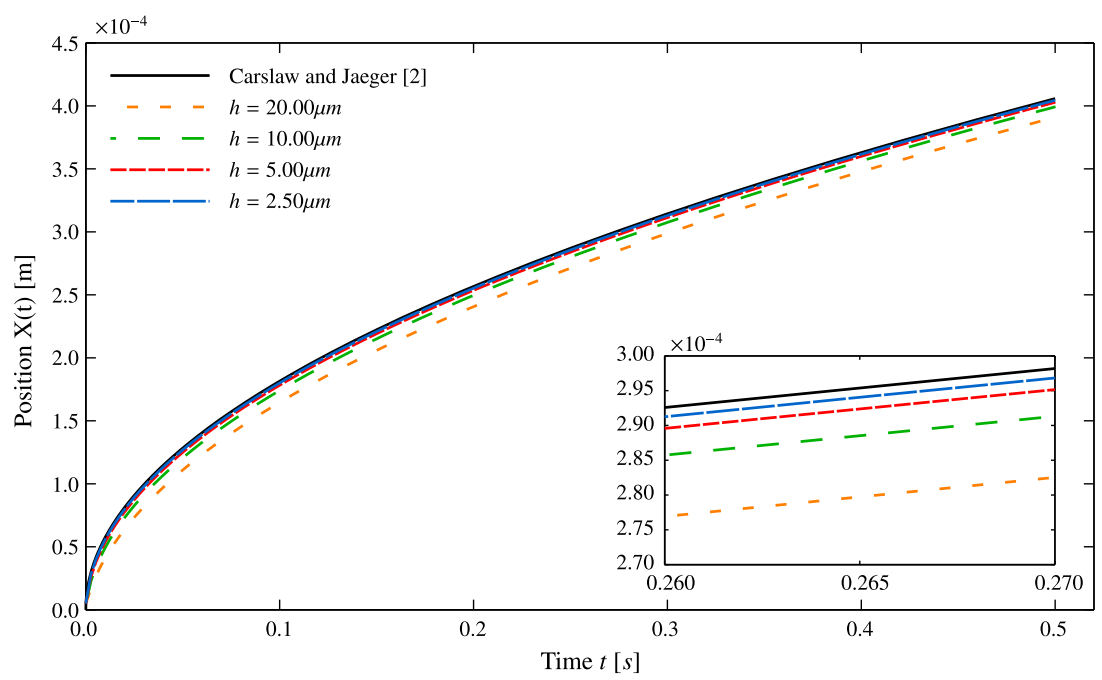

(a) Results obtained by the VOF (FS3D code) method

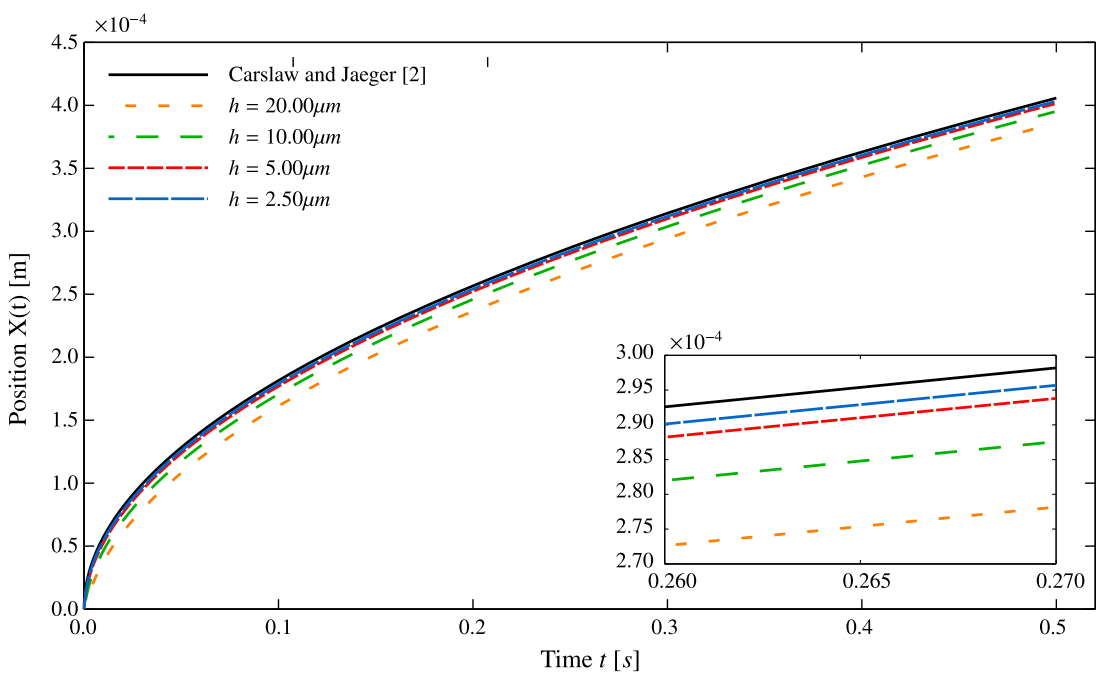

(b) Results obtained by the Level-Set (OpenFOAM ${ }^{\circledR}$ code) method

Fig. 5. Time dependence of the position $X(t)$ of the solid-liquid front plotted for different grid resolutions compared with the analytical solution. The inset magnifies a section of the plot to elucidate the convergence behavior in terms of the grid size.

\subsection{Growth of a parabolic-shaped dendrite}

The second configuration considered in the present work represents the process of formation of a parabolic dendritic pattern. The tip velocity and tip curvature radius of a parabolic dendrite have been investigated experimentally by several authors in the past. Langer and Müller-Krumbhaar [39-41] have performed a stability analysis of dendritic growth rates and developed the so-called 


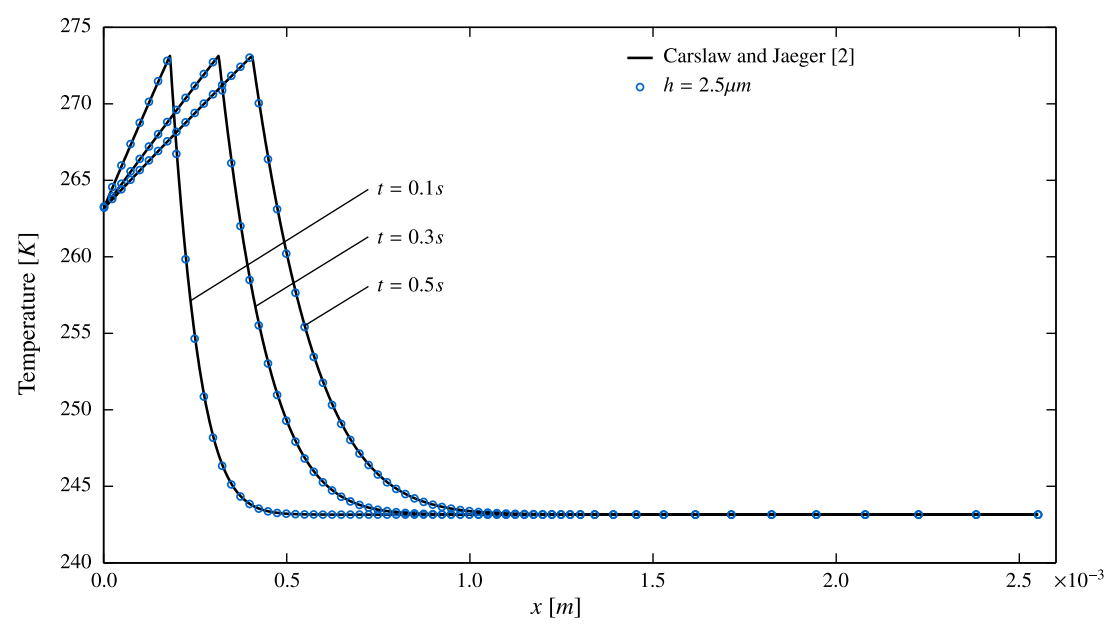

Fig. 6. Spatial distribution of the temperature field at selected time instants $t=0.1 \mathrm{~s}, 0.3 \mathrm{~s}$ and $0.5 \mathrm{~s}$ : results correspond to the VOF (FS3D code) method.

Langer-Müller-Krumbhaar (LM-K) stability theory. They found evidence of a universal law of dendritic growth rates [4], which relates to the dimensionless growth velocity $V$ and the Peclet number $P e$

$V=\sigma^{*} P e^{2}$,

where $P e=v_{t} r_{t} /(2 \alpha)$ and $\sigma^{*}$ is a proportionality constant discussed below. $v_{t}$ and $r_{t}$ denote the tip velocity and tip radius of a dendrite. The physical properties correspond to liquid water. For isothermal, cylindrically symmetric dendrites of paraboloidal shape, Langer et al. [4] state that the Ivantsov solution yields an expression featuring the functional dependency between the Stefan number and the Peclet number

$S t \approx P e \exp (P e) E_{1}(P e)$,

where $E_{1}$ is the exponential integral. For this relation, Langer et al. [4] make the assumption that the capillarity length $d_{0}=c_{p} T_{m} \gamma /\left(\rho L^{2}\right)$, with $\gamma$ denoting the surface tension, is much larger than the radius of curvature $r_{t}$. For the cases presented in this work, $r_{t}$ is at least for a factor of 100 higher than $d_{0}$ within the whole range of supercooling $\Delta T=T_{m}-T_{\infty}$ (varying between $1 \mathrm{~K}$ and $30 \mathrm{~K}$ ). The Stefan number is defined as $S t=c_{p} \Delta T / L$. One further dimensionless number describing this problem is

$\sigma=l d_{0} / r_{t}^{2}$,

where $l=2 \alpha / v_{t}$ is a diffusion length. To determine the tip radius, a stability criterion
$\sigma^{*}=\left(\lambda_{S} /\left(2 \pi r_{t}\right)\right)^{2}$

is used [4], where $\lambda_{S}=2 \pi \sqrt{2 \alpha d_{0} / v_{t}}$ denotes the Mullins and Sekerka [13] stability length. Langer and Müller-Krumbhaar [39] argue that for $\sigma<\sigma^{*}$ the tip becomes linearly unstable and for $\sigma>\sigma^{*}$ the sidebranching instability occurs causing a decrease of $\sigma$. Langer and Müller- Krumbhaar [39] found the following values and variations for $\sigma^{*}$ :

$$
\sigma^{*}= \begin{cases}0.020 \pm 0.007 & (2 \mathrm{D}) \\ 0.025 \pm 0.007 & (3 \mathrm{D})\end{cases}
$$

They represent the values of $\sigma$ at which the dendrite tip grows. This corresponds to the tip radius $r_{t}$ being equal to $\lambda_{s}$. Herewith, the determination of the growth velocity $V$ is enabled and the tip velocity $v_{t}$ can be computed from

$V(S t)=\frac{d_{0} v_{t}}{2 \alpha}$

The tip radius $r_{t}$ is obtained from $P e=v_{t} r_{t} /(2 \alpha)$.

A schematic of the appropriate computational setup is depicted in Fig. 7. The paraboloidal dendrite is initiated in the small region at the lower center of the considered domain meshed by a high resolution grid with equidistantly arranged cells. This fine resolution implies the curvature radius at the tip being resolved with approximately twenty grid cells in order to assure a correct curvature reproduction. Since the curvature radius decreases with

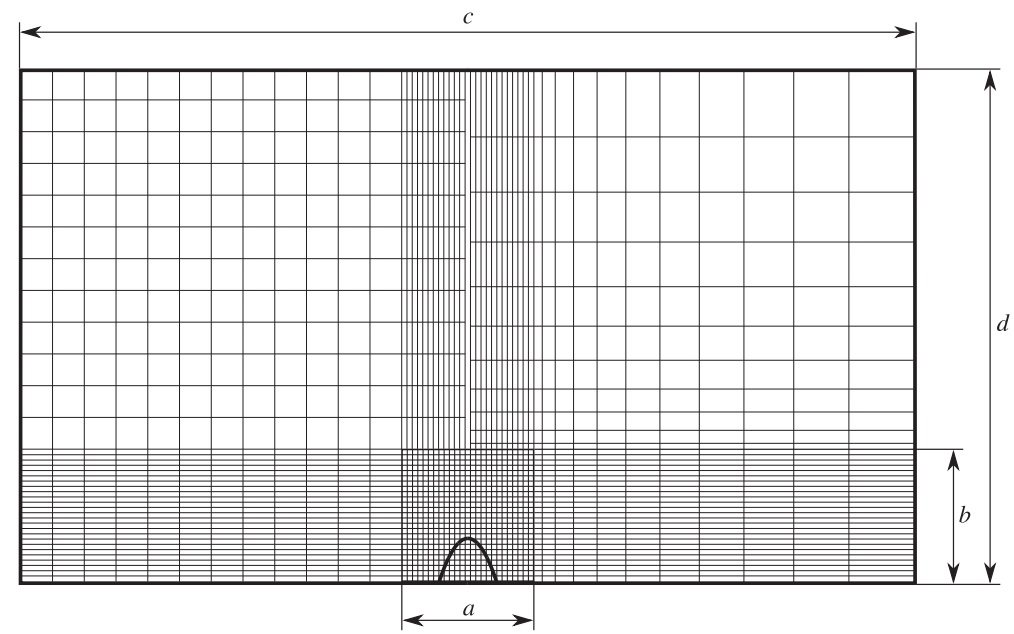

Fig. 7. Schematic of the computational domain (left: the OpenFoam ${ }^{\circledR}$ grid, right: the FS3D grid). 
Table 2

Physical properties of water and ice at $273.15 \mathrm{~K}$.

\begin{tabular}{lllllll}
\hline & $T(\mathrm{~K})$ & $\begin{array}{l}\rho(\mathrm{kg} / \\
\left.\mathrm{m}^{3}\right)\end{array}$ & $\begin{array}{l}L(\mathrm{~kJ} / \\
\mathrm{kg})\end{array}$ & $\begin{array}{l}\sigma(\mathrm{kg} / \\
\left.\mathrm{s}^{2}\right)\end{array}$ & $\begin{array}{l}\lambda(\mathrm{W} / \\
(\mathrm{m} \mathrm{K}))\end{array}$ & $\begin{array}{l}c_{p}(\mathrm{~kJ} / \\
\mathrm{kg} \mathrm{K})\end{array}$ \\
\hline Water & 273.15 & 1000.0 & 333.0 & 0.028 & 0.5624 & 4.218 \\
Ice & 273.15 & 1000.0 & - & - & 2.216 & 2.103 \\
\hline
\end{tabular}

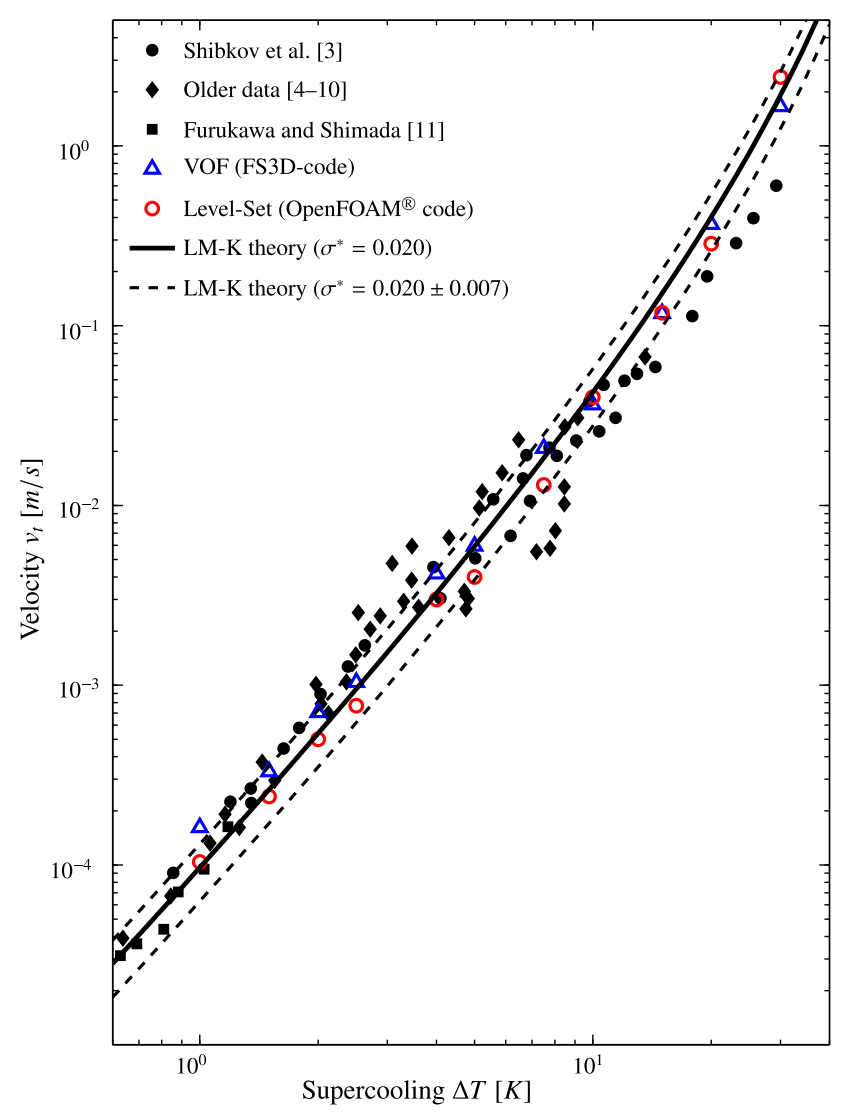

Fig. 8. Tip velocity development as a function of $\Delta T$ : comparison of the results obtained by the VOF and Level-Set methods with available experimental data and theory of Langer and Müller-Krumbhaar [26].

supercooling increase, the grid spacing $h$ is different for every test case. The height of the initial paraboloid is $0.25 b$. Parameter $a$ must be chosen in accordance with the lateral growth of the dendrite, becoming larger for higher supercooling. The boundaries of the computational domain comply with adiabatic conditions; the temperatures at the left, right and upper boundaries must not change during the computation. This is the reason for choosing a somewhat larger computational domain with the dimensions corresponding to $c=21 b$ and $d=10 b$. The computations with the OpenFOAM ${ }^{\circledR}$ code and the Level-Set method have been performed at a coarser grid in the outer domain (displayed on the left of Fig. 7). The computations with the FS3D code applying the VOF method benefited from a certain grid coarsening in the remainder of the computational domain arranged through non-equidistant cells with increasing grid spacing towards the left, right and upper boundaries (displayed on the right of Fig. 7). The dendrite has the initial temperature $T=273.15 \mathrm{~K}$ and the liquid temperature corresponds to that of the respective supercooling. The physical properties of water and ice where both taken at $T=273.15 \mathrm{~K}$, see Table 2 .

The experimental results are taken from the work of Shibkov et al. [3], who presented three result sets: own results, older data
[4-10] and work of Furukawa and Shimada [11]. Fig. 8 shows the tip velocity $v_{t}$ (in $\mathrm{m} / \mathrm{s}$ ) over supercooling $\Delta T$ (in $K$ ). The solid line represents the result obtained by applying the LM-K theory with $\sigma^{*}=0.020$. Additionally, the two dashed lines show the upper and lower limit of the theoretical values of $\sigma^{*}$, cf. Eq. (28). According to Shibkov et al. [3], certain deviations of the theoretical tip velocity from experimental results at higher supercoolings $\Delta T$ might be due to the assumption of small Peclet numbers and non-considering the molecular attachment kinetics.

The temporal extent of the present computations corresponds to the time instant at which the tip curvature starts to deviate from the initially prescribed curvature as predicted by the LM-K theory due to the non-homogeneous distribution of the temperature field in the near-interface region. The Level-Set simulations allow a maximum deviation of $15 \%$ from the initial tip curvature. In FS3D, the tip curvature is overpredicted at the beginning and varies strongly due to the yet extreme temperature gradient at the phase interface. Once, a smooth temperature field has developed, the curvature decreases. The velocity of the tip is determined at the point where the tip curvature equals the curvature of the LM-K theory. At a supercooling of $10 \mathrm{~K}$ and $20 \mathrm{~K}$ the tip curvature converges to $5.9 \%$ and $7.2 \%$ above the LM-K curvature respectively; the tip velocity is determined at the point where this curvature is first reached. The tip velocity developments resulting from the present computations are depicted in Fig. 8. The computationally obtained tip velocities by using both methods (VOF and Level-Set) follow closely the theoretical results and lie within the experimental scatter.

\section{Conclusion}

Two computational model schemes relying on the Volume-ofFluid and Level-Set methods have been successfully applied for investigating the solidification of supercooled water. These schemes have been implemented into the FS3D code and the open source software OpenFOAM ${ }^{\circledR}$, respectively. Excellent agreement between our computations and the theoretical results of Stefan's planar freezing model has been demonstrated. Furthermore, the formation of dendritic patterns has been investigated by performing two-dimensional simulations. The results of dendritic growth exhibit excellent qualitative and quantitative agreement with the marginal stability theory of Langer and Müller-Krumbhaar [39] as well as with the available experiments. Hence, with these preliminary tests both codes were shown to be suited for their respective application in the fundamental research of droplet freezing and airfoil icing as outlined in the introduction.

\section{Acknowledgment}

Financial support of the German Research Council (Deutsche Forschungsgemeinschaft - DFG) through the collaborative research center SFB-TRR 75 is gratefully acknowledged.

\section{References}

[1] Weigand B, Tropea C. Droplet dynamics under extreme boundary conditions: The Collaborative Research Center SFB-TRR 75. In: 12th Triennial international conference on liquid atomization and spray systems ICLASS 2012, Heidelberg, Germany; September 2-6, 2012. <http://www.ilass.uci.edu/>.

[2] Carslaw HS, Jaeger JC. Conduction of heat in solids. Oxford University Press; 1959.

[3] Shibkov AA, Zheltov MA, Korolev AA, Kazakov AA, Leonov AA. Crossover from diffusion-limited to kinetics-limited growth of ice crystals. J Cryst Growth 2005;285:215-27. http://dx.doi.org/10.1016/j.jcrysgro.2005.08.007.

[4] Langer JS, Sekerka RF, Fujioka T. Evidence for a universal law of dendritic growth rates. J Cryst Growth 1978;44:414-8. http://dx.doi.org/10.1016/00220248(78)90007-6.

[5] Ohsaka K, Trinh EH. Apparatus for measuring the growth velocity of dendritic ice in undercooled water. J Cryst Growth 1998;194:138-42. http://dx.doi.org/ $\underline{10.1016 / \mathrm{S} 0022-0248(98) 00661-7 .}$. 
[6] Lindenmeyer CS, Orrok GT, Jackson KA, Chalmers B. Rate of growth of ice crystals in supercooled water. J Chem Phys 1957;27:822-3. http://dx.doi.org/ $10.1063 / 1.1743849$

[7] Hallett J. Experimental studies of the crystallization of supercooled water. J Atmos Sci 1964;21:671-82. http://dx.doi.org/10.1175/15200469(1964)0210671:ESOTCO2.0.CO:2.

[8] Pruppacher HR. On the growth of ice crystals in supercooled water and aqueous solution drops. Pure Appl Geophys 1967;68:186-95. http:// dx.doi.org/10.1007/BF00874894.

[9] Macklin WC, Ryan BF. Growth velocities of ice in supercooled water and aqueous sucrose solutions. Philos Mag 1968;7:83-7. http://dx.doi.org/ $10.1080 / 14786436808218182$

[10] Kallungal JP, Barduhn AJ. Growth rate of an ice crystal in subcooled pure water. AIChE J 1977;23:294-303. http://dx.doi.org/10.1002/aic.690230312.

[11] Furukawa Y, Shimada W. Three-dimensional pattern formation during growth of ice dendrites - its relation to universal law of dendritic growth. J Cryst Growth 1993;128:234-9. http://dx.doi.org/10.1016/0022-0248(93)90325-Q.

[12] Mullins WW, Sekerka RF. Morphological stability of a particle growing by diffusion or heat flow. J Appl Phys 1963;34(2):323-9.

[13] Mullins WW, Sekerka RF. Stability of a planar interface during solidification of a dilute binary alloy. J Appl Phys 1964;35:444-51. http://dx.doi.org/10.1063 1.1713333.

[14] Langer JS. Instabilities and pattern formation in crystal growth. Rev Mod Phys 1980;52(1):1-28. http://dx.doi.org/10.1103/RevModPhys.52.1.

[15] Juric D, Tryggvason G. A front-tracking method for dendritic solidification. J Comput Phys 1996:123:127-48. http://dx.doi.org/10.1006/icph.2001.6726.

[16] Peskin CS. Numerical analysis of blood flow in the heart. J Comput Phys 1977;25:220-52. http://dx.doi.org/10.1016/0021-9991(77)90100-0.

[17] Al-Rawahi N, Tryggvason G. Numerical simulation of dendritic solidification with convection: two-dimensional geometry. J Comput Phys 2002;180(2):471-96. http://dx.doi.org/10.1006/jcph.2002.7092.

[18] Al-Rawahi N, Tryggvason G. Numerical simulation of dendritic solidification with convection: three-dimensional flow. J Comput Phys 2004;194:677-96. http://dx.doi.org/10.1016/j.jcp.2003.09.020.

[19] Udaykumar H, Mittal R, Shyy W. Computation of solid-liquid phase fronts in the sharp interface limit on fixed grids. J Comput Phys 1999;153:535-74. http://dx.doi.org/10.1006/jcph.1999.6294.

[20] Beckermann C, Diepers HJ, Steinbach I, Karma A, Tong X. Modeling melt convection in phase-field simulations of solidification. J Comput Phys 1999;154:468-96. http://dx.doi.org/10.1006/jcph.1999.6323.

[21] Karagadde S, Bhattacharya A, Tomar G, Dutta P. A coupled VOF-IBM-enthalpy approach for modeling motion and growth of equiaxed dendrites in a solidifying melt. J Comput Phys 2012;231:3987-4000. http://dx.doi.org 10.1016/j.jcp.2012.02.001.

[22] Sussman M, Smereka P, Osher S. A level set approach for computing solutions to incompressible two-phase flow. J Comput Phys 1994;114(1):146-59. http:/ dx.doi.org/10.1006/jcph.1994.1155.

[23] Sethian JA. A fast marching level set method for monotonically advancing fronts. Proc Natl Acad Sci USA 1996;93:1591-5. <http://www.pnas.org/ content/93/4/1591.abstract>.

[24] Sethian JA, Strain J. Crystal growth and dendritic solidification. J Comput Phys 1992;98:231-53. http://dx.doi.org/10.1016/0021-9991(92)90140-T.
[25] Chen S, Merriman B, Osher S, Smereka P. A simple level set method for solving Stefan problems. J Comput Phys 1997;135(1):8-29. http://dx.doi.org/10.1006/ icph.1997.5721.

[26] Langer JS, Müller-Krumbhaar H. Stability effects in dendritic crystal growth. J Cryst Growth 1977;42:11-4. http://dx.doi.org/10.1016/0022-0248(77)901713.

[27] Baehr HD, Stephan K. Heat and mass transfer. 2nd ed. Springer-Verlag; 2006

[28] Alexiades V, Solomon A. Mathematical modeling of melting and freezing processes. Hemisphere Publishing Corporation; 1993.

[29] Ferziger JH, Perić M. Computational methods for fluid dynamics. 3rd ed. Springer-Verlag; 2002

[30] Hirt CW, Nichols BD. Volume of fluid (VOF) method for the dynamics of free boundaries. J Comput Phys 1981;39(1):201-25. http://dx.doi.org/10.1016/ 0021-9991(81)90145-5.

[31] Osher S, Sethian JA. Fronts propagating with curvature-dependent speed: algorithms based on Hamilton-Jacobi formulations. J Comput Phys 1988;79(1):12-49. http://dx.doi.org/10.1016/0021-9991(88)90002-2.

[32] Sethian JA. Level set methods: evolving interfaces in geometry, fluid mechanics, computer vision and materials sciences. 1st ed. Cambridge University Press; 1996.

[33] Rider WJ, Kothe DB. Reconstructing volume tracking. J Comput Phys 1998;141(2):112-52. http://dx.doi.org/10.1006/jcph.1998.5906.

[34] Scardovelli R, Zaleski S. Direct numerical simulation of free-surface and interfacial flow. Annu Rev Fluid Mech 1999;31:567-603. http://dx.doi.org/ 10.1146/annurev.fluid.31.1.567.

[35] Rauschenberger P, Schlottke J, Eisenschmidt K, Weigand B. Direct numerical simulation of multiphase flow with rigid body motion in an Eulerian rramework. In: 24th European conference on liquid atomization and spray systems ILASS - Europe 2011, Estoril, Portugal; 2011. <http:// www.ilass.uci.edu/>.

[36] Schlottke J, Rauschenberger P, Weigand B, Ma C, Bothe D. Volume of fluid direct numerical simulation of heat and mass transfer using sharp temperature and concentration fields. In: 24th European conference on liquid atomization and spray systems ILASS - Europe 2011, Estoril, Portugal; 2011. <http://www.ilass.uci.edu/>.

[37] Criscione A, Kintea D, Tuković Ẑ, Jakirlić S, Roisman IV, Tropea C. On computational investigation of the supercooled Stefan problem. In: 12th International conference on liquid atomization and spray systems, Heidelberg, Germany; 2012. <http://www.ilass.uci.edu/>.

[38] Osher S, Fedkiw R. Level set methods and dynamic implicit surfaces. SpringerVerlag; 2003.

[39] Langer JS, Müller-Krumbhaar H. Theory of dendritic growth - I. Elements of a stability analysis. Acta Metall 1978;26:1681-7. http://dx.doi.org/10.1016 0001-6160(78)90078-0.

[40] Langer JS, Müller-Krumbhaar H. Theory of dendritic growth - II. Instabilities in the limit of vanishing surface tension. Acta Metall 1978;26:1689-95. http:/ dx.doi.org/10.1016/0001-6160(78)90079-2.

[41] Langer JS, Müller-Krumbhaar H. Theory of dendritic growth - III. Effects of surface tension. Acta Metall 1978;26:1697-708. http://dx.doi.org/10.1016 0001-6160(78)90080-9. 